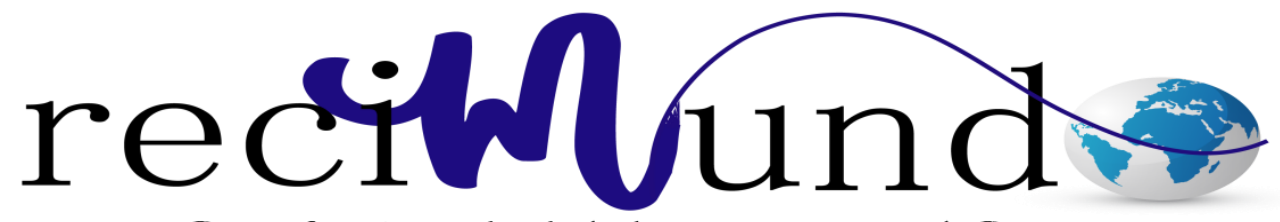

Revista Cientifica Mundo de la Investigación y el Conocimiento

Katherine Jazmín Arévalo Morales a ; Susana de los Ángeles Balarezo Cañar b; Alexandra Silvia Tandazo Lalangui ${ }^{c}$

Morbimortalidad neonatal por anomalías congénitas del tramo gastrointestinal y su relación con antecedentes epidemiológicos prenatales

Revista Científica Mundo de la Investigación y el Conocimiento. Vol. 1 núm., 4, septiembre, 2017, pp. 803-821

DOI: 10.26820/recimundo/1.4.2017.803-821

Editorial Saberes del Conocimiento

a. Hospital del Niño Dr. Francisco Icaza Bustamante; katykatyarevalo@hotmail.com

b. Hospital del Niño Dr. Francisco Icaza Bustamante

c. Hospital del Niño Dr. Francisco Icaza Bustamante 


\section{Morbimortalidad neonatal por anomalías congénitas del tramo gastrointestinal y su relación con antecedentes epidemiológicos prenatales}

Vol. 1, núm. 4., (2017)

Katherine Jazmín Arévalo Morales; Susana de los Ángeles Balarezo Cañar; Alexandra Silvia Tandazo Lalangui

\section{RESUMEN}

Antecedentes.- Las malformaciones congénitas son anomalías en la estructura de un órgano o parte del cuerpo; debido a su desarrollo durante el embarazo, causado por factores genéticos o ambientales, provocando además la interrupción de funcionamiento del órgano afectado su funcionabilidad. $\mathrm{Su}$ importancia radica en ser un problema frecuente (15 de cada 1.000 recién nacidos) y grave, ya que puede comprometer órganos vitales causando muerte y aquellos que sobrevivir, requieren tratamientos largos y costosos. Objetivos.- El objetivo principal de este trabajo ha sido establecer la relación entre los antecedentes epidemiológicos prenatales, tales como factores socioeconómicos, genéticos, maternos, entre otros, y la morbimortalidad por anomalías congénitas del tramo gastrointestinal en neonatos transferidos al Hospital de Niños "Dr. Roberto Gilbert Elizalde" en el periodo de enero 2012 a diciembre de 2013, para lo cual se han determinar la prevalencia de anomalías congénitas del tramo gastrointestinal, las tasas de mortalidad, las características clínicas de los pacientes y los principales antecedentes epidemiológicos prenatales que se han relacionado con la mortalidad por anomalías congénitas. Metodología.- Se trata de un estudio descriptivo observacional, analítico, con un diseño no experimental - retrospectivo, donde se observó la mortalidad de los pacientes con anomalías congénitas. Se utilizó para ello un estudio de casos, en pacientes con anomalías congénitas gastrointestinales. Resultados.- La prevalencia de anomalías congénitas del tramo fue de $23,1 \%$ con un porcentaje de mortalidad de $26,2 \%$ y las tasas de mortalidad para anomalías gastrointestinales de 78,8 por cada 1000 pacientes con anomalías. Conclusión.- Al relacionar con factores de riesgo de mortalidad no se encontró relación con características clínicas mientras que la gastrosquisis presenta un mayor porcentaje de mortalidad, frente a las otras anomalías $(\mathrm{p}=0,035)$.

Palabras clave: Malformaciones congénitas; malformaciones congénitas del tramo grastrointestinal; mortalidad; morbilidad. 


\title{
Morbimortalidad neonatal por anomalías congénitas del tramo gastrointestinal y su relación con antecedentes epidemiológicos prenatales
} Vol. 1, núm. 4., (2017)

Katherine Jazmín Arévalo Morales; Susana de los Ángeles Balarezo Cañar; Alexandra Silvia Tandazo Lalangui

\begin{abstract}
Background: Congenital malformations are abnormalities in the structure of an organ or part of the body; due to its development during pregnancy, caused by genetic or environmental factors, also causing the interruption of functioning of the affected organ its functionability. Its importance is to be a frequent problem (15 out of 1,000 newborns) and serious, since it can compromise vital organs causing death and those that survive, require long and expensive treatments. Objectives.- The main objective of this study was to establish the relationship between prenatal epidemiological antecedents, such as socioeconomic, genetic, maternal factors, among others, and morbidity and mortality due to congenital anomalies of the gastrointestinal tract in neonates transferred to Children's Hospital "Dr . Roberto Gilbert Elizalde "in the period from January 2012 to December 2013, for which the prevalence of congenital anomalies of the gastrointestinal tract, the mortality rates, the clinical characteristics of the patients and the main prenatal epidemiological antecedents have been determined. related to mortality from congenital anomalies. Methodology.- This is a descriptive - observational, analytical study, with a non experimental - retrospective design, where the mortality of patients with congenital anomalies was observed. A case study was used in patients with congenital gastrointestinal abnormalities. Results.- The prevalence of congenital anomalies of the segment was $23.1 \%$ with a mortality rate of $26.2 \%$ and mortality rates for gastrointestinal anomalies of 78.8 per 1000 patients with abnormalities. Conclusion.- When related to risk factors for mortality, no relationship was found with clinical characteristics, whereas gastroschisis had a higher percentage of mortality compared to other abnormalities $(\mathrm{p}=0.035)$.
\end{abstract}

Keywords: Congenital malformations; congenital malformations of the gastrointestinal tract; mortality; morbidity. 


\section{Morbimortalidad neonatal por anomalías congénitas del tramo gastrointestinal y su relación con antecedentes epidemiológicos prenatales}

Vol. 1, núm. 4., (2017)

Katherine Jazmín Arévalo Morales; Susana de los Ángeles Balarezo Cañar; Alexandra Silvia Tandazo Lalangui

\section{Introducción.}

Las anomalías congénitas, las que se conocen con el nombre de defectos de nacimiento, patologías congénitas o malformaciones congénitas, se presentan de forma estructural, o también funcional, tal como se observa en los trastornos metabólicos que se observan desde el nacimiento. (1)

Estás anomalías constituyen un defecto morfológico primario que se origina principalmente durante el proceso de organogenético. Se puede definir como cualquier anormalidad estructural presente en el nacimiento, macroscópica o microscópica, interno o externo, que puede ser debido a causas genéticas, ambientales, o mixtas, o que no se llegan a identificar las causas en la mayoría de los casos (2)

La incidencia de esta patología indica que uno de cada 33 recién nacidos se ven afectados, causando 3,2 millones de discapacidad por año, también se considera que anualmente, aproximadamente 280.000 neonatos fallecen durante las primeras tres semanas de vida por causas relacionadas con anomalías congénitas, (1) por lo que sigue siendo una de las causas más importantes de mortalidad infantil. (3) .Las malformaciones congénitas más comunes son las cardiovasculares y digestivas y las mujeres tienen una mayor tasa de mortalidad. (4)

Junto con la alta tasa de morbilidad, generalmente las malformaciones congénitas presentan un alto costo para la familia y el Estado, debido a los costos relacionados con el tratamiento médico clínico, así como el de rehabilitación, que en la mayoría de los casos no 


\section{Morbimortalidad neonatal por anomalías congénitas del tramo gastrointestinal y su relación con antecedentes epidemiológicos prenatales}

Vol. 1, núm. 4., (2017)

Katherine Jazmín Arévalo Morales; Susana de los Ángeles Balarezo Cañar; Alexandra Silvia

Tandazo Lalangui

resulta ser exitoso, debido a la dificultad en la prevención y las altas tasas de mortalidad, en este punto radica la importancia de las investigaciones científicas sobre esta problemática.

En el Ecuador esta problemática no se encuentra documentada, a pesar de su importancia. Un estudio de Beltrám realizado en Cuenca indicó un porcentaje de Morbilidad de 7,69\% para malformaciones congénitas, sin embargo no registraron la localización de la misma. Una investigación realizada por Salinas en México indicó una tasa de mortalidad de $14.2 \%$ en malformaciones congénitas, donde elaparato gastrointestinal ocupó el segundo lugar (17,3\%), sin embargo este aparato fue el que presentó la mayor tasa de morbilidad $(23,3 \%)$ de los casos totales.

Puesto que la documentación ha resultado ser escasa, se plantea este trabajo que permita aumentar y esclarecer factores relacionados con las malformaciones.

\section{Metodología}

El trabajo que se presenta a continuación es de tipo observacional, considerado los datos estadísticos existentes en el Hospital, además de ser analítico con un enfoque retrospectivo, de corte transversal.

La definición del nivel de investigación es relacional, considerando que se van a establecer las características que se asocian a la mortalidad en pacientes ingresados por anomalías congénitas, donde se usan Odds ratio. Este trabajo tiene un diseño epidemiológico y a su vez analítico. 


\section{Morbimortalidad neonatal por anomalías congénitas del tramo gastrointestinal y su relación con antecedentes epidemiológicos prenatales}

Vol. 1, núm. 4., (2017)

Katherine Jazmín Arévalo Morales; Susana de los Ángeles Balarezo Cañar; Alexandra Silvia Tandazo Lalangui

En este trabajo se observó la mortalidad de los pacientes con anomalías congénitas y se utilizó para ello un estudio de casos considerando la variable defunción.

El universo estuvo constituido por todos los pacientes transferidos al hospital por anomalías congénitas que en total fueron 216, y la muestra la forman todos los pacientes con diagnóstico de anomalías congénitas del tramo gastrointestinal. No se ha considerado realizar un cálculo de la muestra considerando que anualmente se atendieron en el Hospital 65 pacientes por anomalías congénitas gastrointestinales en el periodo descrito, además se considerarán criterios de inclusión y exclusión que se exponen a continuación:

\section{Criterios de inclusión}

Formaron parte del estudio:

- Con diagnóstico de anomalías congénitas del tramo gastrointestinal

- Con edad de nacimiento menor a 7 días

- Que posean información completa

\section{Criterios de exclusión}

Fueron excluidos pacientes:

- que presentaron malformaciones congénitas distintas al tramo gastrointestinal

- que fueron trasladados al hospital después del séptimo día del nacimiento.

- que no posean información completa al momento del levantamiento de la información. 


\section{Morbimortalidad neonatal por anomalías congénitas del tramo gastrointestinal y su relación con antecedentes epidemiológicos prenatales}

Vol. 1, núm. 4., (2017)

Katherine Jazmín Arévalo Morales; Susana de los Ángeles Balarezo Cañar; Alexandra Silvia Tandazo Lalangui

\section{Procedimiento de recolección de la información y técnicas de recolección}

Se utilizó para la recolección de datos el formulario de ficha recolectora diseñado por la autora para el presente estudio, con las variables que guardan directa relación con los objetivos de estudio. Se utilizará como herramientas de consulta las historias clínicas o expedientes clínicos de los años 2012 - 2013 del departamento de Estadísticas del hospital, y se dará apoyo mediante los sistemas informáticos MIS y Servinte.

Se han observado los datos de los pacientes trasferidos, los cuales serán observados hasta que reciban el alta hospitalaria y se produzca el deceso del paciente. Las variables medidas fueron las siguientes considerando puntos de corte:

\section{Tasas de mortalidad}

La tasa de mortalidad considera la cantidad de muertes por cada 1000 pacientes que presenten la patología, para lo cual se ha utilizado la siguiente fórmula: $m x=F \times P x x 1.000$

Dónde:

$\mathrm{Mx}=$ tasa de mortalidad

Fx = Número de pacientes fallecidos por anomalías congénitas del tramo gastrointestinalPx $=$ Número total de pacientes con anomalías gastrointestinales .

Se establecieron tres tasas de mortalidad, la primera relacionada con anomalías gastrointestinales, la segunda con anomalías generales y la tercera con todos los ingresos. 


\section{Morbimortalidad neonatal por anomalías congénitas del tramo gastrointestinal y su relación con antecedentes epidemiológicos prenatales}

Vol. 1, núm. 4., (2017)

Katherine Jazmín Arévalo Morales; Susana de los Ángeles Balarezo Cañar; Alexandra Silvia Tandazo Lalangui

\section{Técnicas de análisis estadístico}

Los datos fueron recogidos en un hoja de cálculo de Microsoft Excel 2013 @ para luego ser analizados con el paquete estadístico SPSS 21 (IBM ®). A las variables cualitativas se les aplicó CHI2 para indicar significancia estadística (se considerarán valores de $\mathrm{p}<0,05$ para un 95\% de confianza), para medir la relación se aplicara Odds ratio e intervalos de confianza con el 95\% de confianza, donde se han formado 2 grupos en relación con la mortalidad asociándolo con las características clínicas, epidemiológicas, ginecológicas entre otras. Los datos se han presentados en cuadros y gráficos estadísticos para una mejor comprensión.

\section{Resultados}

Se observó una prevalencia de 23,1\% (65/281) de pacientes transferidos por anomalías congénitas del tramo gastrointestinal, frente a otras malformaciones en el Hospital de niños Dr. Roberto Gilbert Elizalde, durante el 2012 -2013. (Tabla 1)

\begin{tabular}{|c|c|c|}
\hline Pacientes & Frecuencia & Prevalencia \\
\hline Transferidos por otras anomalias congénitas & 216 & 76,9 \\
\hline Con anomalías congénitas gastrointestinales & 65 & 23,1 \\
\hline Total de pacientes con anomalías congénitas & 281 & 100,0 \\
\hline
\end{tabular}

Tabla 1. Prevalencia de anomalías del tracto gastrointestinal 


\section{Morbimortalidad neonatal por anomalías congénitas del tramo gastrointestinal y su relación con antecedentes epidemiológicos prenatales}

Vol. 1, núm. 4., (2017)

Katherine Jazmín Arévalo Morales; Susana de los Ángeles Balarezo Cañar; Alexandra Silvia

Tandazo Lalangui

Al clasificar estas malformaciones se pudo apreciar que la más frecuente fue el ano imperforado 41,5\% (27/65), seguida por Gastrosquisis 20,0\% (13/65) y la artresia esofágica 15,4\%, (10/65) con una significancia estadística de $\mathrm{p}<0,001$, la Artresia duodenal presentó un 7,7\% (5/65). (Tabla 2)

Se puedo apreciar que el 29,2\% (19/65) de los pacientes presentó múltiples malformaciones congénitas, donde 3 pacientes presentaron ano imperforado + Fístula rectouretral y Atresia esofágica + Fístula traqueosofágica como las principales asociaciones (Tabla 2)

\begin{tabular}{|c|c|c|c|}
\hline Clasificación de las malformaciones & $\begin{array}{c}\text { Frecuencia } \\
(n=65)\end{array}$ & Porcentaje & $\mathrm{p}$ \\
\hline Ano anterior & 1 & 1,5 & $<0,001$ \\
\hline Atresia Esofágica & 10 & 15,4 & \\
\hline Distribución intestinal alta & 4 & 6,2 & \\
\hline Gastrosquisis & 13 & 20 & \\
\hline Ano imperforado & 27 & 41,5 & \\
\hline Otras & 10 & 15,4 & \\
\hline Atresia Duodenal & 5 & 50,0 & \\
\hline Malrotación intestinal & 1 & 10,0 & \\
\hline Bandas periduodenales & 1 & 10,0 & \\
\hline Membrana pilórica & 1 & 10,0 & \\
\hline Enfermedad de Hirshprung & 1 & 10,0 & \\
\hline Hernia diafragmática & 1 & 10,0 & \\
\hline Múltiples malformaciones & 19 & 29,2 & \\
\hline
\end{tabular}

Tabla 2. Clasificación de anomalías del tracto gastrointestinal

El porcentaje de mortalidad se sitúa en el 26,2\% (17/65), con una tasa de 261 x 1000 en pacientes con enfermedades congénitas del tramo gastrointestinal, 78,8 x 1000 para todas las 


\section{Morbimortalidad neonatal por anomalías congénitas del tramo gastrointestinal y su relación con antecedentes epidemiológicos prenatales}

Vol. 1, núm. 4., (2017)

Katherine Jazmín Arévalo Morales; Susana de los Ángeles Balarezo Cañar; Alexandra Silvia Tandazo Lalangui

malformaciones congénitas y 9,5 x 1000 para todos las transferencias hospitalarias durante el periodo de estudio. (Tabla 3)

La distribución de la mortalidad por sexo indica una mayor prevalencia para hombres $(58,8 \%, 10 / 17)$ que para mujeres $(41,20 \%, 7 / 17)$, sin embargo no se observan diferencias significativas entre los grupos $(\mathrm{p}=0.127)$ donde la tasa de mortalidad para pacientes de sexo masculino fue de 153 x 1000 y para mujeres de 107 x 1000. (Tabla 3)

\begin{tabular}{|c|c|c|c|c|c|c|}
\hline Mortalidad & Frecuencia & Porcentaje & $\begin{array}{c}\text { Tasa } x \\
\text { enfermedad } \\
\text { congénita del } \\
\text { tramo } \\
\text { gastrointestinal }\end{array}$ & $\begin{array}{c}\text { Tasa } x \\
\text { enfermedades } \\
\text { congénitas }\end{array}$ & $\begin{array}{c}\text { Tasa } \mathrm{x} \\
\text { ingresos } \\
\text { hospitalarios }\end{array}$ & \\
\hline $\mathrm{Si}$ & 17 & 26,2 & 261,5 & 78,7 & 9,50783 & \\
\hline No & 48 & 73,8 & & & & \\
\hline \multicolumn{2}{|c|}{ Sexo } & Mortalidad & $\begin{array}{l}\text { Tasa de } \\
\text { mortalidad }\end{array}$ & $p$ & RR & IC \\
\hline \multirow[t]{2}{*}{ Masculino } & $f(x)$ & 10 & 153,8 & 0,127 & 2,381 & $\begin{array}{c}(0,77- \\
7,36) \\
\end{array}$ \\
\hline & $\%$ & $58,80 \%$ & & & & \\
\hline \multirow[t]{2}{*}{ Femenino } & $f(x)$ & 7 & 107,7 & & & \\
\hline & $\%$ & $41,20 \%$ & & & & \\
\hline \multicolumn{2}{|c|}{ Edad gestacional } & Mortalidad & $\begin{array}{c}\text { Tasa de } \\
\text { mortalidad }\end{array}$ & $p$ & RR & IC \\
\hline \multirow[t]{2}{*}{ Pretérmino } & $f(x)$ & 6 & 92,3 & 0,318 & 1,83 & $\begin{array}{c}(0,55- \\
6,09)\end{array}$ \\
\hline & $\%$ & $35,30 \%$ & & & & \\
\hline \multirow[t]{2}{*}{ A término } & $f(x)$ & 11 & 169,2 & & & \\
\hline & $\%$ & $64,70 \%$ & & & & \\
\hline $\begin{array}{l}\text { Mortalidad } \\
\text { antes de } \\
\text { los 7dias }\end{array}$ & 12 & 18,5 & & & & \\
\hline
\end{tabular}

Tabla 3. Tasas de mortalidad de anomalías del tracto gastrointestinal y características clínicas 


\section{Morbimortalidad neonatal por anomalías congénitas del tramo gastrointestinal y su relación con antecedentes epidemiológicos prenatales}

Vol. 1, núm. 4., (2017)

Katherine Jazmín Arévalo Morales; Susana de los Ángeles Balarezo Cañar; Alexandra Silvia

Tandazo Lalangui

Al observar la mortalidad por edad gestacional se pudo apreciar una tasa disminuida para el 35,3\% (6/17) para pacientes Pretérmino y 64,7\% (11/17), para parto atérmino; con tasas del 92 x 1000 Pretérmino y 169 x 1000 a término, sin significancia estadística, p=0,318. (Tabla 8). El 18,5\% (12/65) de los pacientes murió antes de los 7 primeros días de vida. (Tabla 3) Las características clínicas de los pacientes que presentaron malformaciones congénitas indican que según la edad gestacional, el 26,2\% (17/65) fueron pretérmino, con un mayor porcentaje de pacientes de sexo femenino $(56,9 ; 37 / 65)$ frente a los de sexo masculino $(43,1 \% ; 28 / 65)$. Existió un predominio de ser el primer hijo $(26,2 \% ; 17 / 65)$ y el gripo etario más frecuente para edad de la madre fue de 18 - $30(53,8 \% ; 35 / 65)$ seguido de 31-40 (30,8\%; 20/65). Existió un claro predominio para pacientes con procedencia fuera de la ciudad de Guayaquil (Rural) 76,9\% (50/65), mientras que el 41,5\% de las madres tenía instrucción secundaria y el 56,9\% (37/65) estuvieron dedicadas a los quehaceres domésticos. (Tabla 4)

Los hábitos tóxicos, que podrían estar relacionados con las anomalías congénitas han indicado que el 35,4\% (23/65) tenían problemas con el alcohol, mientras que solo el 7,7\% (5/65) de las madres presentaron tal condición, valores similares se registraron para tabaquismo, donde el 36,9\% (24/65) de los padres presentaron esta condición. Solo en el caso de uno de los pacientes resultó que el padre consumía drogas. (Tabla 4) .Solo el 3,1\% (2/65) presentó diabetes, Hipertensión arterial presente en el 15,4\% (10/65) (Tabla 4)

Con respecto a la exposición a agentes tóxicos se aprecia que un 13,8\% (9/65) de los pacientes estuvo expuesto a pesticidas, 9,2\% (6/65) a fármacos y 24,6\% (16/65) a productos químicos. (Tabla 4) 


\section{Morbimortalidad neonatal por anomalías congénitas del tramo gastrointestinal y su relación con antecedentes epidemiológicos prenatales}

Vol. 1, núm. 4., (2017)

Katherine Jazmín Arévalo Morales; Susana de los Ángeles Balarezo Cañar; Alexandra Silvia Tandazo Lalangui

\begin{tabular}{|c|c|c|}
\hline Caracteristica & Frecuencia / Media & Porcentaje / DS* \\
\hline \multicolumn{3}{|l|}{ Edad Gestacional } \\
\hline Pretérmino & 17 & 26,2 \\
\hline A têrmino & 48 & 73,8 \\
\hline \multicolumn{3}{|l|}{ Sexo } \\
\hline Masculino & 28 & 43,1 \\
\hline Femenino & 37 & 56,9 \\
\hline \multicolumn{3}{|l|}{ Edad de la madre } \\
\hline Menos de 18 & 5 & 7,7 \\
\hline $18-30$ & 35 & 53,8 \\
\hline $31-40$ & 20 & 30,8 \\
\hline Más de 40 & 3 & 4,6 \\
\hline Sin registros & 2 & 3,1 \\
\hline \multicolumn{3}{|l|}{ Procedencia } \\
\hline Urbano & 13 & 20 \\
\hline Rural & 50 & 76,9 \\
\hline Sin registros & 2 & 3,1 \\
\hline \multicolumn{3}{|l|}{ Ocupación } \\
\hline Quehaceres domésticos & 37 & 56,9 \\
\hline Agricultura / Obrera & 5 & 7,7 \\
\hline Secretaria / cajera & 3 & 4,6 \\
\hline Estudiante & 3 & 4,6 \\
\hline Trabajadora sexual & 1 & 1,5 \\
\hline No especifica & 16 & 24,6 \\
\hline \multicolumn{3}{|l|}{ Alcoholismo } \\
\hline Madre & 5 & 7,7 \\
\hline Padre & 23 & 35,4 \\
\hline Ambos & 3 & 4,6 \\
\hline Ninguno & 34 & 52,3 \\
\hline \multicolumn{3}{|l|}{ Tabaquismo } \\
\hline Madre & 2 & 3,1 \\
\hline Padre & 24 & 36,9 \\
\hline Ambos & 1 & 1,5 \\
\hline Ninguno & 38 & 58,5 \\
\hline \multicolumn{3}{|l|}{ Drogas } \\
\hline Padre & 1 & 1,5 \\
\hline Ninguno & 64 & 98,5 \\
\hline Diabetes & 2 & 3,1 \\
\hline Hipertensión arterial & 10 & 15,4 \\
\hline Pesticidas & 9 & 13,8 \\
\hline Fármacos & 6 & 9,2 \\
\hline Productos Quimicos & 16 & 24,6 \\
\hline
\end{tabular}

Tabla 4. Características clínicas, ginecológicas, hábitos tóxicos, infecciones, otras complicaciones y exposición a agentes contaminantes 


\section{Morbimortalidad neonatal por anomalías congénitas del tramo gastrointestinal y su relación con antecedentes epidemiológicos prenatales}

Vol. 1, núm. 4., (2017)

Katherine Jazmín Arévalo Morales; Susana de los Ángeles Balarezo Cañar; Alexandra Silvia Tandazo Lalangui

El conjunto de patologías asociadas a esta enfermedad, que estuvieron presentes en el 41,3\% (28/65) de los pacientes de las que destacan Síndrome de Down $(15,4 \% ; 10 / 65)$ y la comunicación intrauricular $(4,6 \% ; 3 / 65)$

Luego para continuar con la investigación se relacionaron las principales características observadas con la mortalidad y se obtuvieron los siguientes resultados:

Para madres menores de 18 años se pudo apreciar un mayor porcentaje de mortalidad al compararla con el grupo de niños que no murieron $(11,8 \% ; 2 / 17$ y $6,50 \% ; 3 / 46)$ sin embargo no existió diferenciassignificativas entre los grupos ( $\mathrm{p}=0,255$; tabla 6) Tampoco se observó diferencia entre la procedencia del paciente (Tabla 6)

Se pudo apreciar en la tabla 6, que existe una asociación entre el tipo de anomalía y la mortalidad, siendo la gastrosquisis la que presenta un mayor porcentaje de mortalidad $(47,10 \%$; 8/17 y 10,40\% 5/48) p=0,035.Tampoco se pudo establecer una relación entre el alcoholismo y tabaquismo con las anomalías congénitas. (Tabla 6) 


\section{Morbimortalidad neonatal por anomalías congénitas del tramo gastrointestinal y su relación con antecedentes epidemiológicos prenatales}

Vol. 1, núm. 4., (2017)

Katherine Jazmín Arévalo Morales; Susana de los Ángeles Balarezo Cañar; Alexandra Silvia Tandazo Lalangui

\begin{tabular}{|c|c|c|c|c|c|c|c|}
\hline \multicolumn{8}{|l|}{ Edad de la madre } \\
\hline \multirow[t]{2}{*}{ Menos de 18} & $f(x)$ & 2 & 3 & 5 & 0,255 & $\mathrm{na}^{*}$ & na* \\
\hline & $\%$ & $11,80 \%$ & $6,50 \%$ & $7,90 \%$ & & & \\
\hline \multirow[t]{2}{*}{$18-30$} & $f(x)$ & 12 & 23 & 35 & & & \\
\hline & $\%$ & $70,60 \%$ & $50,00 \%$ & $55,60 \%$ & & & \\
\hline \multirow[t]{2}{*}{$31-40$} & $f(x)$ & 2 & 18 & 20 & & & \\
\hline & $\%$ & $11,80 \%$ & $39,10 \%$ & $31,70 \%$ & & & \\
\hline \multirow[t]{2}{*}{ Más de 40} & $f(x)$ & 1 & 2 & 3 & & & \\
\hline & $\%$ & $5,90 \%$ & $4,30 \%$ & $4,80 \%$ & & & \\
\hline \multicolumn{8}{|l|}{ Procedencia } \\
\hline \multirow[t]{2}{*}{ Urbano } & $f(x)$ & 4 & 9 & 13 & 0,617 & 1,4 & $0,367-5,04$ \\
\hline & $\%$ & $25,00 \%$ & $19,10 \%$ & $20,60 \%$ & & & \\
\hline \multirow[t]{2}{*}{ Rural } & $f(x)$ & 12 & 38 & 50 & & & \\
\hline & $\%$ & $75,00 \%$ & $80,90 \%$ & $79,40 \%$ & & & \\
\hline \multicolumn{8}{|l|}{$\begin{array}{l}\text { Clasificación de la } \\
\text { malformación }\end{array}$} \\
\hline \multirow[t]{2}{*}{ Ano anterior } & $f(x)$ & 0 & 1 & 1 & 0,035 & na* & na* \\
\hline & $\%$ & $0,00 \%$ & $2,10 \%$ & $1,50 \%$ & & & \\
\hline \multirow[t]{2}{*}{ Atrésia Esofágica } & $f(x)$ & 3 & 7 & 10 & & & \\
\hline & $\%$ & $17,60 \%$ & $14,60 \%$ & $15,40 \%$ & & & \\
\hline \multirow{2}{*}{$\begin{array}{l}\text { Distribución } \\
\text { intestinal alta }\end{array}$} & $f(x)$ & 1 & 3 & 4 & & & \\
\hline & $\%$ & $5,90 \%$ & $6,30 \%$ & $6,20 \%$ & & & \\
\hline \multirow[t]{2}{*}{ Gastrosquitis } & $f(x)$ & 8 & 5 & 13 & & & \\
\hline & $\%$ & $47.10 \%$ & $10.40 \%$ & $2000 \%$ & & & \\
\hline \multirow[t]{2}{*}{ Ano imperforado } & $f(x)$ & 4 & 23 & 27 & & & \\
\hline & $\%$ & $23,50 \%$ & $47,90 \%$ & $41,50 \%$ & & & \\
\hline \multirow[t]{2}{*}{ Otras } & $f(x)$ & 1 & 9 & 10 & & & \\
\hline & $\%$ & $5,90 \%$ & $18,80 \%$ & $15,40 \%$ & & & \\
\hline \multicolumn{8}{|l|}{ Alcoholismo } \\
\hline \multirow[t]{2}{*}{ Madre } & $f(x)$ & 2 & 3 & 5 & 0,309 & na* & na* \\
\hline & $\%$ & $11,80 \%$ & $6,30 \%$ & $7,70 \%$ & & & \\
\hline \multirow[t]{2}{*}{ Padre } & $f(x)$ & 6 & 17 & 23 & & & \\
\hline & $\%$ & $35,30 \%$ & $35,40 \%$ & $35,40 \%$ & & & \\
\hline \multirow[t]{2}{*}{ Ambos } & $f(x)$ & 2 & 1 & 3 & & & \\
\hline & $\%$ & $11,80 \%$ & $2,10 \%$ & $4,60 \%$ & & & \\
\hline Ninguno & $f(x)$ & 7 & 27 & 34 & & & \\
\hline & $\%$ & $41,20 \%$ & $56,30 \%$ & $52,30 \%$ & & & \\
\hline Tabaquismo & & & & & & & \\
\hline Madre & $f(x)$ & 1 & 1 & 2 & 0,759 & na* & na" \\
\hline & $\%$ & $5,90 \%$ & $2,10 \%$ & $3,10 \%$ & & & \\
\hline Padre & $f(x)$ & 7 & 17 & 24 & & & \\
\hline & $\%$ & $41,20 \%$ & $35,40 \%$ & $36,90 \%$ & & & \\
\hline Ambos & $f(x)$ & 0 & 1 & 1 & & & \\
\hline & $\%$ & $0,00 \%$ & $2,10 \%$ & $1,50 \%$ & & & \\
\hline Ninguno & $f(x)$ & 9 & 29 & 38 & & & \\
\hline & $\%$ & $52,90 \%$ & $60,40 \%$ & $58,50 \%$ & & & \\
\hline No & $f(x)$ & 12 & 36 & 48 & & & \\
\hline & $\%$ & $70,60 \%$ & $75,00 \%$ & $73,80 \%$ & & & \\
\hline $\begin{array}{l}\text { Patologias } \\
\text { asociadas }\end{array}$ & & & & & & & \\
\hline $\mathrm{Si}$ & $f(x)$ & 8 & 20 & 28 & 0,7 & 1,244 & $0,409-3,783$ \\
\hline & $\%$ & $47,10 \%$ & $41,70 \%$ & $43,10 \%$ & & & \\
\hline No & $f(x)$ & 9 & 28 & 37 & & & \\
\hline & $\%$ & $52,90 \%$ & $58,30 \%$ & $56,90 \%$ & & & \\
\hline
\end{tabular}

Tabla 6. Relación entre las características de los pacientes y las anomalías congénitas 


\section{Morbimortalidad neonatal por anomalías congénitas del tramo gastrointestinal y su relación con antecedentes epidemiológicos prenatales}

Vol. 1, núm. 4., (2017)

Katherine Jazmín Arévalo Morales; Susana de los Ángeles Balarezo Cañar; Alexandra Silvia Tandazo Lalangui

\section{Discusión}

La prevalencia de pacientes transferidos por anomalías congénitas del tramo gastrointestinal fue de 23,1\% (65/281), frente a otras malformaciones en el Hospital de niños Dr. Roberto Gilbert Elizalde, durante el 2012 -2013, esta tasa resultó ser similar a la expuesta por Salinas en México en las que determinó un porcentaje de 27\% para tales anomalías, en una causística de 256 neonatos dentro de los primeros 28 día de nacimiento. (7)

Por otro lado Navarrete en México, indicó que la prevalencia de malformaciones gastrointestinales solo en el 4,4\% de los pacientes, resultado un valor bastante discorde con lo encontrado aquí. (8) Chang en Guatemala indicó una prevalencia de 21,9\%, lo que vuelve a tener relación con lo expresado en este trabajo. (9)

Se pudo observar que la anomalía más frecuente fue el ano imperforado 41,5\% (27/65), seguida por Gastrosquisis 20,0\% (13/65) y la atresia esofágica $15,4 \%$, (10/65), lo que vuelve a coincidir con lo expuesto por Chang, el cual indica que las anomalías principales fueron las anorrectales $(24.48 \%)$ y los defectos de la pared abdominal (24.48\%), a esto debe agregárseles las anomalías esofágicas con un porcentaje de $18.18 \%$. (9) Mientras que en otro estudio la principal fue malrotación intestinal (17,18\%), enfermedad de Hirschsprung(13,64\%), divertículo de Meckel $(13,14 \%)$ atresia de esófago $(12,13 \%)$ entre las principales. Se puede apreciar que el 29,2\% (19/65) de los pacientes presentó múltiples malformaciones congénitas, este valor se encuentra por debajo de lo descrito por Enriquez, quien describió que el 45,3\% de los pacientes con anomalías congénitas digestivas tuvieron presentaciones múltiples. (10) 


\section{Morbimortalidad neonatal por anomalías congénitas del tramo gastrointestinal y su relación con antecedentes epidemiológicos prenatales}

Vol. 1, núm. 4., (2017)

Katherine Jazmín Arévalo Morales; Susana de los Ángeles Balarezo Cañar; Alexandra Silvia Tandazo Lalangui

El porcentaje de mortalidad se puede indicar en el 26,2\% (17/65), con una tasa de $261 \mathrm{x}$ 1000 en pacientes con enfermedades congénitas del tramo gastrointestinal, 78,8 x 1000 para todas las malformaciones congénitas y 9,5 x 1000 para todos las transferencias hospitalarias durante el periodo de estudio.

Salinas indicó un porcentaje de mortalidad para anomalías gastrointestinales de 17,3\%. (7) y Chang un porcentaje de $48 \%$.

En Colombia la tasa de mortalidad por malformaciones congénitas se mantuvo constante durante años con un promedio de 66,8 defunciones por 10.000 habitantes, sin que se especifique la tasa por cada una, según lo expone González. (11) Mientras que en un estudio realizado en México, la tasa de mortalidad fue de 16,6 defunción por cada nacido vivo. (12)

Al considerar las características se observó una mayor prevalencia de pacientes de sexo femenino $(56,9 ; 37 / 65)$, el grupo etario más frecuente para edad de la madre fue de 18 - 30 (53,8\%; 35/65, el 35,4\% (23/65) de los padres tenían problemas con el alcohol, mientras que solo el 7,7\% (5/65) de las madres presentaron tal condición. Solo el 1,2\% Diabetes Mellitus tipo 2, además un 10,61\% de los pacientes estuvo expuesto a fármacos. (9)

Se pudo observar que la anomalía más frecuente fue el ano imperforado 41,5\% (27/65), seguida por Gastrosquisis 20,0\% (13/65) y la atresia esofágica 15,4\%, (10/65), lo que vuelve a coincidir con lo expuesto por Chang, el cual indica que las anomalías principales fueron las anorrectales $(24.48 \%)$ y los defectos de la pared abdominal $(24.48 \%)$, a esto debe agregárseles las anomalías esofágicas con un porcentaje de 18.18\%. (9) Mientras que en otro estudio la 


\section{Morbimortalidad neonatal por anomalías congénitas del tramo gastrointestinal y su relación con antecedentes epidemiológicos prenatales}

Vol. 1, núm. 4., (2017)

Katherine Jazmín Arévalo Morales; Susana de los Ángeles Balarezo Cañar; Alexandra Silvia Tandazo Lalangui

principal fue malrotación intestinal $(17,18 \%)$, enfermedad de Hirschsprung(13,64\%), divertículo de Meckel $(13,14 \%)$ atresia de esófago $(12,13 \%)$ entre las principales.

Se puede apreciar que el 29,2\% (19/65) de los pacientes presentó múltiples malformaciones congénitas, este valor se encuentra por debajo de lo descrito por Enriquez, quien describió que el 45,3\% de los pacientes con anomalías congénitas digestivas tuvieron presentaciones múltiples. (10)

El porcentaje de mortalidad se puede indicar en el 26,2\% (17/65), con una tasa de $261 \mathrm{x}$ 1000 en pacientes con enfermedades congénitas del tramo gastrointestinal, 78,8 x 1000 para todas las malformaciones congénitas y 9,5 x 1000 para todos las transferencias hospitalarias durante el periodo de estudio.

Salinas indicó un porcentaje de mortalidad para anomalías gastrointestinales de 17,3\%. (7) y Chang un porcentaje de $48 \%$.

En Colombia la tasa de mortalidad por malformaciones congénitas se mantuvo constante durante años con un promedio de 66,8 defunciones por 10.000 habitantes, sin que se especifique la tasa por cada una, según lo expone González. (11) Mientras que en un estudio realizado en México, la tasa de mortalidad fue de 16,6 defunción por cada nacido vivo. (12)

Al considerar las características se observó una mayor prevalencia de pacientes de sexo femenino $(56,9 ; 37 / 65)$, el grupo etario más frecuente para edad de la madre fue de 18 - 30 $(53,8 \% ; 35 / 65$, el $35,4 \%$ (23/65) de los padres tenían problemas con el alcohol, mientras que solo 


\section{Morbimortalidad neonatal por anomalías congénitas del tramo gastrointestinal y su relación con antecedentes epidemiológicos prenatales}

Vol. 1, núm. 4., (2017)

Katherine Jazmín Arévalo Morales; Susana de los Ángeles Balarezo Cañar; Alexandra Silvia Tandazo Lalangui

el 7,7\% (5/65) de las madres presentaron tal condición. Solo el 1,2\% Diabetes Mellitus tipo 2, además un 10,61\% de los pacientes estuvo expuesto a fármacos. (9)

\section{Bibliografía.}

1. OMS. Anomalías congénitas. [Online].; 2012 [cited 2014. Available from: http://www.who.int/mediacentre/factsheets/fs370/es/index.html

2. Herrera Y. Comportamiento de la mortalidad fetal e infantil por malformaciones congénitas. Isla de la Juventud. 2006-2011. REMIJ. 2013; 14(1): p. 1-14.

3. Hernandez J. Mortalidad perinatal por defectos estructurales congénitos: un estudio de sitio. Perinatol Reprod Hum. 2007;: p. 125-132.

4. Pino M. Diagnóstico prenatal de gastrosquisis. Presentación de un caso. Mediciego. 2012;: p. $1-3$.

5. Montes C. Características generales de la atención a recién nacidos con malformaciones congénitas en un hospital infantil. Medisan. 2010;: p. 1-7.

6. Quiroz V. El diagnóstico prenatal de cardiopatías congénitas mejora el pronóstico neonatal. Rev Chil Obstet Ginecol. 2006;: p. 267-273.

7. Salinas V. Malformaciones congénitas como causa de hospitalización en una Unidad de Terapia Intensiva Neonatal. Perinatol. Reprod. Hum. 2012 Abril - junio; 26(2): p. 83-89. 


\section{Morbimortalidad neonatal por anomalías congénitas del tramo gastrointestinal y su relación con antecedentes epidemiológicos prenatales}

Vol. 1, núm. 4., (2017)

Katherine Jazmín Arévalo Morales; Susana de los Ángeles Balarezo Cañar; Alexandra Silvia

Tandazo Lalangui

8. Navarrete E. Prevalencia de malformaciones congénitas registradas en el certificado de nacimiento y de muerte fetal. méxico, 2009-2010. Bol Med Hosp Infant Mex. 2013; 70(6): p. 499-505.

9. Chang H. Caracterización epidemiológica de anomalías congénitas gastrointestinales Guatemala: Universidad de San Carlos de Guatemala; 2013.

10. Enríquez E. Malformaciones digestivas y su asociación a patologíasindrómica y defectos genéticos. Cir Pediatr. 2010; 23: p. 46-52.

11. González M. Protocolo de vigilancia y control de anomalías congénitas: Instituto Nacional de Salud; 2010.

12. Gómez A. La mortalidad infantil por malformaciones congénitas en México: un problema de oportunidad y acceso al tratamiento. Rev Panam Salud Publica. 2008 Noviembre; 24(5). 